Noname manuscript No.

(will be inserted by the editor)

\title{
Local deterministic simulation of equatorial Von Neumann measurements on tripartite GHZ state
}

\author{
Arup Roy · Amit Mukherjee · Some \\ Sankar Bhattacharya - Manik Banik . \\ Subhadipa Das
}

Received: date / Accepted: date

\begin{abstract}
Experimental free-will or measurement independence is one of the crucial assumptions in derivation of any nonlocal theorem. Any nonlocal correlation obtained in quantum world can have a local deterministic explanation if there is no experimental free-will in choosing the measurement settings. Recently, in [Phys. Rev. Lett. 105, 250404 (2010)] it has been shown that to obtain a local deterministic description for singlet state correlation one does not need to give up measurement independence completely, but a partial measurement dependence suffices. In three party scenario considering GHZ correlation one can exhibit absolute contradiction between quantum theory and local realism. In this paper we show that such correlation also has local deterministic description if measurement independence is given up, even if not completely. We provide a local deterministic model for equatorial Von

\footnotetext{
Arup Roy

Physics and Applied Mathematics Unit, Indian Statistical Institute, 203 B.T. Road, Kolkata700108, India

E-mail: arup145.roy@gmail.com

Amit Mukherjee

Physics and Applied Mathematics Unit, Indian Statistical Institute, 203 B.T. Road, Kolkata700108, India

E-mail: amitisiphys@gmail.com

Some Sankar Bhattacharya

Physics and Applied Mathematics Unit, Indian Statistical Institute, 203 B.T. Road, Kolkata700108, India

E-mail: somesankar@gmail.com

Manik Banik

Physics and Applied Mathematics Unit, Indian Statistical Institute, 203 B.T. Road, Kolkata700108, India

E-mail: manik11ju@gmail.com

Subhadipa Das

S.N. Bose National Center for Basic Sciences, Block JD, Sector III, Salt Lake, Kolkata700098, India.

E-mail: sbhdpa.das@bose.res.in
} 
Neumann measurements on tripartite GHZ state by sacrificing measurement independence partially.

\section{Introduction}

One of the most surprising features of quantum mechanics is that it exhibits nonlocal correlations i.e. measurements performed on several quantum systems in an entangled state may contain correlations in their outcomes that cannot be simulated by shared local variables. Though the observation that quantum theory predicts nonlocal correlations goes all the way back to the famous EPR argument [1], it is the remarkable Bell's theorem in 1964 [2] which established that the conflict between quantum theory and local realism could be experimentally decided and this theorem motivated successful experimental tests in this regard $[3,4,5]$. Whereas tests of Bell's inequality only demonstrate the said contradictions in a statistical manner, it was the scientist-trio Greenberger, Horne and Zeilinger who first pointed out that considering more than two particles it is possible to demonstrate absolute contradiction between the predictions of local realism and those of quantum mechanics [6] which is experimentally verified in [7]. Other than this foundational interest the study of nonlocal correlation has been boosted in the last decade from information theoretic perspective (see [8] for review on Bell nonlocality). It has been proved that nonlocal correlations play important role in "device independent" quantum key distribution $[9,10,11,12]$ and random number generation $[13,14,15]$. Thus quantification of the amount of nonlocality in a correlation demands interest from both the foundational as well as practical perspectives.

One practical measure to quantify the amount of nonlocality has been introduced in the Refs. $[16,17,18]$. They pointed out that the amount of communication that is required (either in the worst case scenario or on average), in addition to shared randomness (a local resource), in order to simulate the behavior of entangled quantum systems could be a good measure of nonlocality. For local correlations, no communication is needed, as shared randomness suffice; they thus have a "communication measure" of nonlocality equal to zero. For the bipartite scenario one of the most important result in this regard has been proved by Toner and Bacon, who showed that for the case of local projective measurements on an entangled Bell state exact simulation is possible using local hidden variables augmented by just one bit of classical communication [19]. In multi-party scenario investigation of such simulation protocol for Greenberger-Horne-Zeilinger (GHZ) state has been started in [20, 21]. Branciard and Gisin proved that 3 classical bits (in total) turn out to be sufficient to simulate all equatorial Von Neumann measurements on the tripartite GHZ state [22]. Recently, Brassard et al [23] have shown that the GHZ joint discrete probability distributions can also be simulated even under the random bit model, in which one is only allowed to access an unbiased IID source. 
On the other hand in the derivation of any nonlocal theorem one crucial assumption is measurement independence: that measurement settings can be chosen independent of any underlying variables describing the system. Though in [24] Shimony et al. have emphasized the reasonableness of this postulate, in recent time Hall $[25,26]$ and Barrett and Gisin [27] independently studied this assumption more explicitly. In [28], Brans gave an explicit local and deterministic model for correlations between any two spin- $1 / 2$ particles where an underlying random variable fully determines not only the joint measurement outcomes, but also the associated measurement settings, i.e. there is no measurement independence at all. Interestingly, introducing a suitable measure of the degree of measurement independence, a possible candidate to quantify the amount of nonlocality, Hall showed that one does not need to relax measurement independence fully to obtain a no-signaling and deterministic model of the singlet state rather only $14 \%$ relaxation suffices [25].

In this paper we consider 3-qubit GHZ quantum correlations and present no-signaling and deterministic protocol to simulate such nonlocal correlations by relaxing degree of measurement independence, partially. This problem is the straightforward next step after the 2-qubit singlet state. We show that $71.5 \%$ relaxation of the degree of measurement independence is sufficient to obtain a no-signaling and deterministic model for all equatorial Von Neumann measurements on the 3-qubit GHZ state. Note that relaxation of measurement independence is higher for the GHZ state in comparison to the singlet state which is not counterintuitive as refutation of EPR argument by considering GHZ state is strikingly more direct than singlet state. Interestingly, we also find that to reproduce the expectation value for all equatorial Von Neumann measurements on the 3-qubit GHZ state one does not need to relax $71.5 \%$ measurement independence, rather only $62.5 \%$ relaxation is sufficient.

The paper is organized in the following way. In Section (2) we briefly discuss the GHZ correlation and also the simulation protocol of equatorial GHZ correlation as introduced by Branciard and Gisin. In Section (3) we present our result i.e measurement dependent but no-signaling and deterministic simulation protocols for all equatorial Von Neumann measurements on the tripartite GHZ state. Section (4) contains discussions and conclusions.

\section{Tripartite GHZ correlation}

GHZ state was first introduced in [6]. The authors of [6] and then Mermin in [29] showed that the refutation of EPR argument by GHZ state is strikingly more direct than the one Bell's theorem provides for Bohm's version of EPR. The refutation is not only stronger it is no longer statistical and can be accomplished in a single run. Besides this foundational importance GHZ state exhibits various applications in information theoretic processes, for example quantum secret sharing [30], entanglement broadcasting [31], simultaneous quantum secure direct communication [32] etc. 
The form of three-qubit GHZ state held among three parties, say Alice, Bob and Charlie, looks:

$$
|\psi\rangle_{G H Z}=\frac{1}{\sqrt{2}}(|000\rangle+|111\rangle)
$$

Here $|0\rangle(|1\rangle)$ represents the eigenvector of the Pauli Z operator with eigenvalue $+1(-1)$. Let the three parties perform spin measurements along the direction $\hat{m}_{A}, \hat{m}_{B}$ and $\hat{m}_{C}$, respectively; where the measurement direction for the party $X(=A, B, C)$ is specified by the block vector $\hat{m}_{X} \equiv\left(\sin \theta_{X} \cos \phi_{X}, \sin \theta_{X}\right.$ $\left.\sin \phi_{X}, \cos \theta_{X}\right)$, with $\theta_{X} \in[0, \pi]$ and $\phi_{X} \in[0,2 \pi]$. Denoting measurement outcome as $a, b$ and $c$ respectively, where $a, b, c \in[+1,-1]$, the three party GHZ correlation can be expressed in the following form:

$$
\begin{aligned}
P\left(a b c \mid\left\{\hat{m}_{X}\right\}\right)= & \frac{1}{8}\left[1+a b \cos \theta_{A} \cos \theta_{B}\right. \\
& +b c \cos \theta_{B} \cos \theta_{C}+c a \cos \theta_{C} \cos \theta_{A} \\
& +a b c \cos \theta_{A} \cos \theta_{B} \cos \theta_{C} \\
& +a b c \sin \theta_{A} \sin \theta_{B} \sin \theta_{C} \\
& \left.\cos \left(\phi_{A}+\phi_{B}+\phi_{C}\right)\right]
\end{aligned}
$$

where $P\left(a b c \mid\left\{\hat{m}_{X}\right\}\right) \equiv P\left(a b c \mid \hat{m}_{A}, \hat{m}_{B}, \hat{m}_{C}\right)$ is the probability of obtaining outcome $a, b$ and $c$ by Alice, Bob and Charlie, respectively, when they perform measurements $\hat{m}_{A}, \hat{m}_{B}$ and $\hat{m}_{C}$ on their respective parts of the shared GHZ state. If the measurement directions for all the parties are chosen from an equatorial plane (i.e $\sin \theta_{A}=\sin \theta_{B}=\sin \theta_{C}=0$ ) then Eqn.(2) becomes:

$$
P\left(a b c \mid\left\{\hat{m}_{X}\right\}\right)=\frac{1}{8}\left[1+a b c \cos \left(\phi_{A}+\phi_{B}+\phi_{C}\right)\right] .
$$

In this case the expectation value becomes:

$$
\begin{aligned}
\left\langle\hat{m}_{A} \hat{m}_{B} \hat{m}_{C}\right\rangle & =\sum_{a, b, c} a b c P\left(a b c \mid \hat{m}_{A}, \hat{m}_{B}, \hat{m}_{C}\right) \\
& =\cos \left(\phi_{A}+\phi_{B}+\phi_{C}\right)
\end{aligned}
$$

while all single and bipartite marginals vanish. Note that although the choice of equatorial measurements is restrictive, these are enough to come up with the "GHZ paradox".

Branciard- Gisin simulation protocol

In [22], Branciard and Gisin provided a simulation protocol for the correlation

(4) with bounded communications among the parties. They first showed that with 3 bits of classical communication ( 2 bits from Bob to Alice and 1 bit from 
Charlie to Alice) supplemented by several shared randomness and also local variables, Alice, Bob and Charlie can simulate the following statistics:

$$
E_{1}(\phi)=1-\frac{2 \phi-\sin 2 \phi}{\pi} \text { for } \phi \in[0, \pi]
$$

Of course Branciard-Gisin(BG) simulation protocol provides vanishing single and bipartite marginals. Moreover correlation $E_{1}(\phi)$ is stronger than the desired $\cos \phi$ correlation in the sense that $\left|E_{1}(\phi)\right| \geq|\cos \phi|$, for all $\phi$ (see Fig.1). They then show that mixing the correlations of the form $E((2 m+1) \phi)$, with

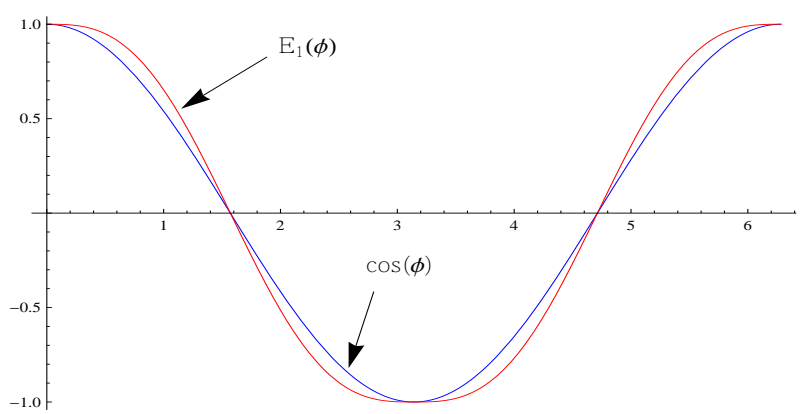

Fig. 1 (Color on-line). Correlation $E_{1}(\phi)$ is the BG correlation whereas $\cos (\phi)$ is the desired GHZ correlation. This figure is taken from [22].

$m \in Z$, one can obtain the desired $\cos \phi$ correlation as $E((2 m+1) \phi)$ will preserve the perfect (anti-)correlations for $\phi=0$ and $\pi$. The mixing is done in the following way

$$
\cos \phi=\sum_{m \geq 0} p_{2 m+1} E((2 m+1) \phi)
$$

with $p_{2 m+1} \geq 0$ for all $m \geq 0$. In particular, for $\phi=0$ one gets $\sum_{m \geq 0} p_{2 m+1}=$ 1 .

\section{Measurement dependence simulation protocol}

In this section we provide a simulation protocol for the equatorial GHZ correlation with reduced measurement independence. Before providing the simulation protocol we briefly describe measurement (in)dependence.

In simulation of nonlocal correlation, the parties involved are allowed to hold pre-shared variables, say $\lambda \in \Lambda, \Lambda$ being the space of shared variable(s) or the hidden variable(s). The assumption of measurement independence demands that distribution of the underlying variable is independent of the measurement settings, i.e.,

$$
p\left(\lambda \mid\left\{\hat{m}_{X}\right\}\right)=p\left(\lambda \mid\left\{\hat{m}_{X}^{\prime}\right\}\right) .
$$


Where $\hat{m}_{X}$ and $\hat{m}_{X}^{\prime}$ are two different measurement settings for the party $X$. In the BG simulation Alice and Bob hold shared variables but distribution of these variables do not depend on the measurement settings - satisfying the measurement independence assumption. Measurement independence is often justified by the notion of experimental free will, i.e., that experimenters can freely choose between different measurement settings irrespective of the underlying variable $\lambda$ describing the system.

The degree to which an underlying model violates measurement independence is most simply quantified by the variational distance [25]:

$$
M:=\sup _{\left\{\hat{m}_{X}\right\},\left\{\hat{m}_{X}^{\prime}\right\}} \int d \lambda\left|p\left(\lambda \mid\left\{\hat{m}_{X}\right\}\right)-p\left(\lambda \mid\left\{\hat{m}_{X}^{\prime}\right\}\right)\right|
$$

with $0 \leq M \leq 2$. Clearly, a distance of $M=0$ corresponds to the case of full measurement independence as per Eqn.(7), consistent with maximum experimental free will in choosing measurement settings. Conversely, suppose that $M$ attains its greatest possible value, $M=2$, for some model. Hence, there are at least two particular joint measurement settings, $\left\{\hat{m}_{X}\right\}$ and $\left\{\hat{m}_{X}^{\prime}\right\}$, such that for any $\lambda$ at most one of these settings is possible. Hence, no experimental free will whatsoever can be exercised to choose between these settings.

The fraction of measurement independence corresponding to a given model is defined by $[25,26]$ :

$$
F:=1-\frac{M}{2}
$$

with $0 \leq F \leq 1$, where $F=0$ corresponds to the case where no experimental free will can be enjoyed to choose measurement settings and $F=1$ corresponds to complete experimental free will. Any value for $F$ strictly lying in between 0 and 1 corresponds to the case where experimenter's free will in choosing the measurement settings is restricted. Note that, geometrically, $F$ also represents the minimum degree of overlap between any two underlying distributions $p\left(\lambda \mid\left\{\hat{m}_{X}\right\}\right.$ and $p\left(\lambda \mid\left\{\hat{m}_{X}^{\prime}\right\}\right.$.

\subsection{Simulation protocol}

Let Alice, Bob and Charlie share a variable $\boldsymbol{\lambda}$ chosen from unit circle. Now given a measurement direction from equatorial plane Alice, Bob and Charlie give their answers in the following way:

$$
\begin{aligned}
& A\left(\hat{m}_{A}, \boldsymbol{\lambda}\right)=\operatorname{sign}\left(\hat{m}_{A} \cdot \boldsymbol{\lambda}\right), \\
& B\left(\hat{m}_{B}, \boldsymbol{\lambda}\right)=\operatorname{sign}\left(\hat{m}_{B} \cdot \boldsymbol{\lambda}\right), \\
& C\left(\hat{m}_{C}, \boldsymbol{\lambda}\right)=\operatorname{sign}\left(\hat{m}_{C} \cdot \boldsymbol{\lambda}\right),
\end{aligned}
$$

where

$$
\operatorname{sign}(\alpha)=s(\alpha):=\left\{\begin{array}{l}
1 \text { if } \alpha \geq 0 \\
-1 \text { otherwise }
\end{array}\right.
$$


The distribution of the variable $\boldsymbol{\lambda}$ is not uniform, rather the distribution depends on measurement directions of Alice, Bob and Charlie. The distribution is given by:

$$
\begin{aligned}
\rho\left(\boldsymbol{\lambda} \mid\left\{\hat{m}_{X}\right\}\right): & =\rho^{\prime}\left(\boldsymbol{\lambda} \mid\left\{\hat{m}_{X}\right\}\right) \Theta\left(\phi_{A B}-\phi_{A C}\right) \\
& +\rho^{\prime \prime}\left(\boldsymbol{\lambda} \mid\left\{\hat{m}_{X}\right\}\right) \Theta\left(\phi_{A C}-\phi_{A B}\right)
\end{aligned}
$$

where $\Theta$ is the step function defined as:

$$
\Theta(\alpha)= \begin{cases}1 & \text { if } \alpha \geq 0 \\ 0 & \text { otherwise }\end{cases}
$$

$\phi_{A B}$ : angle between measurement directions of Alice and Bob;

$\phi_{A C}$ : angle between measurement directions of Alice and Charlie;

$$
\begin{aligned}
\rho^{\prime}\left(\boldsymbol{\lambda} \mid\left\{\hat{m}_{X}\right\}\right):= & \frac{1+\beta \cos \left(\phi_{A}+\phi_{B}+\phi_{C}\right)}{8\left(\pi-\phi_{A B}\right)} \\
& \text { if } s\left(\hat{m}_{A} \cdot \boldsymbol{\lambda}\right)=s\left(\hat{m}_{B} \cdot \boldsymbol{\lambda}\right)=s\left(\hat{m}_{C} \cdot \boldsymbol{\lambda}\right)=\beta \\
:= & \frac{1+\beta \cos \left(\phi_{A}+\phi_{B}+\phi_{C}\right)}{8\left(\phi_{A B}-\phi_{A C}\right)} \\
& \text { if }-s\left(\hat{m}_{A} \cdot \boldsymbol{\lambda}\right)=-s\left(\hat{m}_{B} \cdot \boldsymbol{\lambda}\right)=s\left(\hat{m}_{C} \cdot \boldsymbol{\lambda}\right)=\beta \\
:= & \frac{1+\beta \cos \left(\phi_{A}+\phi_{B}+\phi_{C}\right)}{8 \phi_{A B}} \\
& \text { if } s\left(\hat{m}_{A} \cdot \boldsymbol{\lambda}\right)=-s\left(\hat{m}_{B} \cdot \boldsymbol{\lambda}\right)=-s\left(\hat{m}_{C} \cdot \boldsymbol{\lambda}\right)=\beta \\
:= & \frac{1+\beta \cos \left(\phi_{A}+\phi_{B}+\phi_{C}\right)}{8} \delta\left(\boldsymbol{\lambda}-\beta \boldsymbol{\lambda}_{0}\right) \\
& \text { if }-s\left(\hat{m}_{A} \cdot \boldsymbol{\lambda}\right)=s\left(\hat{m}_{B} \cdot \boldsymbol{\lambda}\right)=-s\left(\hat{m}_{C} \cdot \boldsymbol{\lambda}\right)=\beta
\end{aligned}
$$

with $\beta \in\{+1,-1\}$; and

$$
\begin{aligned}
\rho^{\prime \prime}\left(\boldsymbol{\lambda} \mid\left\{\hat{m}_{X}\right\}\right):= & \frac{1+\beta \cos \left(\phi_{A}+\phi_{B}+\phi_{C}\right)}{8\left(\pi-\phi_{A C}\right)} \\
& \text { if } s\left(\hat{m}_{A} \cdot \boldsymbol{\lambda}\right)=s\left(\hat{m}_{B} \cdot \boldsymbol{\lambda}\right)=s\left(\hat{m}_{C} \cdot \boldsymbol{\lambda}\right)=\beta \\
:= & \frac{1+\beta \cos \left(\phi_{A}+\phi_{B}+\phi_{C}\right)}{8} \delta\left(\boldsymbol{\lambda}-\beta \boldsymbol{\lambda}_{0}\right) \\
& \text { if }-s\left(\hat{m}_{A} \cdot \boldsymbol{\lambda}\right)=-s\left(\hat{m}_{B} \cdot \boldsymbol{\lambda}\right)=s\left(\hat{m}_{C} \cdot \boldsymbol{\lambda}\right)=\beta \\
:= & \frac{1+\beta \cos \left(\phi_{A}+\phi_{B}+\phi_{C}\right)}{8 \phi_{A C}} \\
& \text { if } s\left(\hat{m}_{A} \cdot \boldsymbol{\lambda}\right)=-s\left(\hat{m}_{B} \cdot \boldsymbol{\lambda}\right)=-s\left(\hat{m}_{C} \cdot \boldsymbol{\lambda}\right)=\beta \\
:= & \frac{1+\beta \cos \left(\phi_{A}+\phi_{B}+\phi_{C}\right)}{8\left(\phi_{A C}-\phi_{A B}\right)} \\
& \text { if }-s\left(\hat{m}_{A} \cdot \boldsymbol{\lambda}\right)=s\left(\hat{m}_{B} \cdot \boldsymbol{\lambda}\right)=-s\left(\hat{m}_{C} \cdot \boldsymbol{\lambda}\right)=\beta
\end{aligned}
$$


$\lambda_{0}$ be a fixed vector chosen from the unit circle and $\delta$ be the Dirac delta function. Given the value assignment as Eqn.(10) and the distribution of the hidden variable $\boldsymbol{\lambda}$ as Eqn.(11), it is easy to check that the quantum probability distribution of Eqn.(3) is obtained by integrating out the variable $\boldsymbol{\lambda}$, i.e.,

$$
P\left(a b c \mid\left\{\hat{m}_{X}\right\}\right)=\int d \boldsymbol{\lambda} p(a b c \mid \boldsymbol{\lambda}) \rho\left(\boldsymbol{\lambda} \mid\left\{\hat{m}_{X}\right\}\right),
$$

where $p(a b c \mid \boldsymbol{\lambda})=\delta_{a, A\left(\hat{m}_{A}, \boldsymbol{\lambda}\right)} \delta_{b, B\left(\hat{m}_{B}, \boldsymbol{\lambda}\right)} \delta_{c, C\left(\hat{m}_{C}, \boldsymbol{\lambda}\right)}$. The amount of degree of measurement dependence for the above simulation protocol turns out to be $M \simeq 1.43$ and hence $F \simeq 28.5 \%$ (Appendix A). Thus to obtain a deterministic, no-signaling model for the equatorial Von Neumann measurements on tripartite GHZ state one does not need to give up complete experimental freewill, rather $71.5 \%$ lack of measurement independence is sufficient. Note that, if in some simulation protocol of a correlation the fraction of measurement independence takes a value $0<f<1$, it does not imply that there exist a simulation model that replicates the corresponding correlation as long as one can have a dependent model at least $1-f$ percent of the time but must have an independent model the other fraction $f$ of time. This is because the definition of $F$ lacks such an operational interpretation.

It is noteworthy that given an expectation value the probability distribution associated with it is not unique. Interestingly we find that if one wants to reproduce the expectation value of the equatorial measurements on GHZ state i.e. Eqn.(4) but does not bother about reproducing the quantum probabilities (i.e Eqn.(3)) then there exists a simulation model where the amount of experimental free-will that has to be sacrificed is $62.5 \%$ (Appendix B).

\section{Discussion and conclusion}

In this paper we provide a protocol based upon relaxation of measurement independence which can reproduce the equatorial Von Neumann measurements results on three qubits GHZ deterministically and unlike ref.[22] our simulation protocol uses no classical communications among the parties. This result tells that if sufficient amount of free-will is not assured for the involved parties to chose their measurement settings then GHZ argument cannot reveal the conflict between quantum theory and local realism. In [26], the author has shown that EPR-Kochen-Specker theorem due to Mermin [29] fails if measurement independence is relaxed by $33.3 \%$. Please note that, neither any local deterministic simulation protocol for GHZ state has been given in [26] nor it is known whether $33.3 \%$ relaxation of measurement independence is sufficient for modeling equatorial Von Neumann measurements statistics locally and deterministically. Thus optimality of our model is a question of further investigation. It is also interesting to extend this model to all measurements (which in our case is restricted to equatorial Von Neumann measurements).

In the bipartite scenario it has been shown in [33] that it is possible to construct a local deterministic simulation protocol for singlet correlation by 


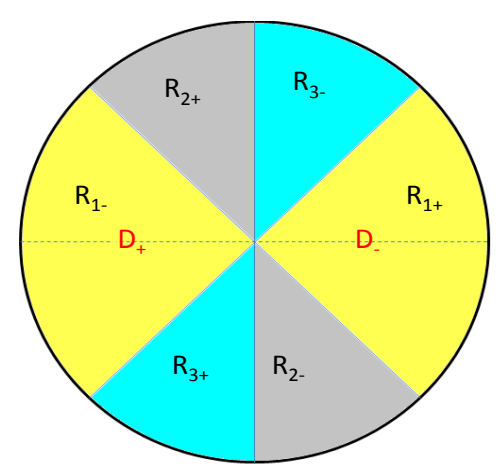

Fig. 2 (Color on-line). $R_{1+}\left(R_{1-}\right)$ is the region where $s\left(\hat{m}_{A} \cdot \boldsymbol{\lambda}\right)=s\left(\hat{m}_{B} \cdot \boldsymbol{\lambda}\right)=s\left(\hat{m}_{C} \cdot \boldsymbol{\lambda}\right)=$ $+1(-1) ; R_{2+}\left(R_{2-}\right)$ is the region where $s\left(\hat{m}_{A} \cdot \boldsymbol{\lambda}\right)=-s\left(\hat{m}_{B} \cdot \boldsymbol{\lambda}\right)=-s\left(\hat{m}_{C} \cdot \boldsymbol{\lambda}\right)=+1(-1)$ and $R_{3+}\left(R_{3-}\right)$ is the region where $-s\left(\hat{m}_{A} \cdot \boldsymbol{\lambda}\right)=-s\left(\hat{m}_{B} \cdot \boldsymbol{\lambda}\right)=s\left(\hat{m}_{C} \cdot \boldsymbol{\lambda}\right)=+1(-1) . D_{+}\left(D_{-}\right)$ denotes the fixed vector $\boldsymbol{\lambda}_{\mathbf{0}}\left(-\boldsymbol{\lambda}_{\mathbf{0}}\right)$

sacrificing measurement independence of one party whereas the other party enjoys complete free-will. In reduced measurement independence simulation of GHZ correlation it is also interesting to study whether such a model is possible where some (and not all ) parties sacrifice measurement independence and rest enjoy complete free-will.

\section{A Appendix A: Calculation of measurement dependency}

The degree of measurement dependency is quantified as Eqn.(8). To find $M$ we have to maximize the difference between the densities of hidden variable (HV) for all pairs of measurement settings over the HV space. The difference will be maximized if the upper value of the distribution of $\mathrm{HV}$ corresponding to one measurement setting overlaps by maximum amount with the lower value of the other measurement setting. The distribution of $\mathrm{HV}$ for each measurement setting consists of three pair of regions comprising two opposite spherical sectors $\left(\left\{R_{1+}, R_{1-}\right\},\left\{R_{2+}, R_{2-}\right\},\left\{R_{3+}, R_{3-}\right\}\right)$ and a pair of delta functions $\left(D_{+}, D_{-}\right)$as defined in Eqn.(13)and shown in Fig.2. The distribution of HV for measurement setting $\left\{\hat{m}_{X}\right\}$ and $\left\{\hat{m}_{X}^{\prime}\right\}$ are denoted by prime and unprimed region, respectively (see Fig.3). The maximum of right hand side of Eqn.(8) occurs when $R_{1+}$ contains $D_{-}^{\prime}$ and the region $R_{3-}^{\prime}$ completely and the region $R_{2-}^{\prime}$ partially as shown in Fig.3. It becomes that $M=1.43$ and thus we have $F:=1-\frac{M}{2} \simeq 28.5 \%$.

\section{B Appendix B: Simulation of GHZ expectation value}

In this case also Alice, Bob and Charlie share a variable $\boldsymbol{\lambda}$ chosen from unit circle and given a measurement direction from equatorial plane Alice, Bob and 


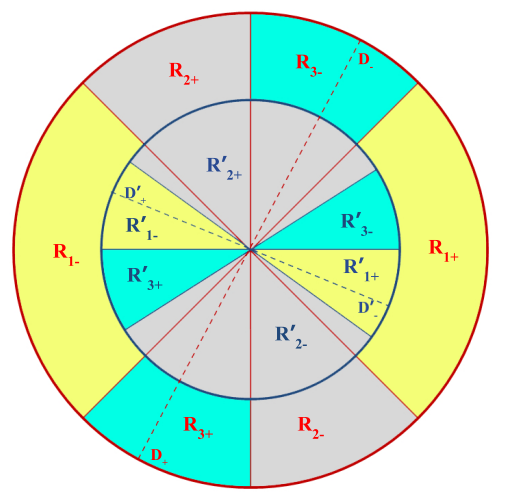

Fig. 3 (Color on-line). This figure shows distribution of HV corresponding to measurement settings $\left\{\hat{m}_{X}\right\}$ and $\left\{\hat{m}_{X}^{\prime}\right\}$. The inner circle represents the distribution corresponding to the measurement setting $\left\{\hat{m}_{X}^{\prime}\right\}$ and the outer circle corresponding to the measurement setting $\left\{\hat{m}_{X}\right\}$. The maximum of the right hand side of Eqn.(8) occurs when $R_{1+}$ contains $D_{-}^{\prime}$ and the region $R_{3-}^{\prime}$ completely and the region $R_{2-}^{\prime}$ partially.

Charlie give there answer like Eqn.(10). The distribution of the variable $\boldsymbol{\lambda}$ in this case is given by

$$
\begin{aligned}
\vartheta\left(\boldsymbol{\lambda} \mid\left\{\hat{m}_{X}\right\}\right):=\vartheta^{\prime}\left(\boldsymbol{\lambda} \mid\left\{\hat{m}_{X}\right\}\right) \Theta\left(\phi_{A B}-\phi_{A C}\right) \\
+\vartheta^{\prime \prime}\left(\boldsymbol{\lambda} \mid\left\{\hat{m}_{X}\right\}\right) \Theta\left(\phi_{A C}-\phi_{A B}\right)
\end{aligned}
$$

where

$$
\begin{aligned}
\vartheta^{\prime}\left(\boldsymbol{\lambda} \mid\left\{\hat{m}_{X}\right\}\right):= & \frac{1+\beta \cos \left(\phi_{A}+\phi_{B}+\phi_{C}\right)}{6\left(\pi-\phi_{A B}\right)} \\
& \text { if } s\left(\hat{m}_{A} \cdot \boldsymbol{\lambda}\right)=s\left(\hat{m}_{B} \cdot \boldsymbol{\lambda}\right)=s\left(\hat{m}_{C} \cdot \boldsymbol{\lambda}\right)=\beta \\
:= & \frac{1+\beta \cos \left(\phi_{A}+\phi_{B}+\phi_{C}\right)}{6\left(\phi_{A B}-\phi_{A C}\right)} \\
& \text { if }-s\left(\hat{m}_{A} \cdot \boldsymbol{\lambda}\right)=-s\left(\hat{m}_{B} \cdot \boldsymbol{\lambda}\right)=s\left(\hat{m}_{C} \cdot \boldsymbol{\lambda}\right)=\beta \\
:= & \frac{1+\beta \cos \left(\phi_{A}+\phi_{B}+\phi_{C}\right)}{6 \phi_{A B}} \\
& \text { if } s\left(\hat{m}_{A} \cdot \boldsymbol{\lambda}\right)=-s\left(\hat{m}_{B} \cdot \boldsymbol{\lambda}\right)=-s\left(\hat{m}_{C} \cdot \boldsymbol{\lambda}\right)=\beta
\end{aligned}
$$

with $\beta \in\{+1,-1\}$; and

$$
\begin{aligned}
\vartheta^{\prime \prime}\left(\boldsymbol{\lambda} \mid\left\{\hat{m}_{X}\right\}\right):= & \frac{1+\beta \cos \left(\phi_{A}+\phi_{B}+\phi_{C}\right)}{6\left(\pi-\phi_{A C}\right)} \\
& \text { if } s\left(\hat{m}_{A} \cdot \boldsymbol{\lambda}\right)=s\left(\hat{m}_{B} \cdot \boldsymbol{\lambda}\right)=s\left(\hat{m}_{C} \cdot \boldsymbol{\lambda}\right)=\beta \\
:= & \frac{1+\beta \cos \left(\phi_{A}+\phi_{B}+\phi_{C}\right)}{6 \phi_{A C}} \\
& \text { if } s\left(\hat{m}_{A} \cdot \boldsymbol{\lambda}\right)=-s\left(\hat{m}_{B} \cdot \boldsymbol{\lambda}\right)=-s\left(\hat{m}_{C} \cdot \boldsymbol{\lambda}\right)=\beta
\end{aligned}
$$




$$
\begin{aligned}
:= & \frac{1+\beta \cos \left(\phi_{A}+\phi_{B}+\phi_{C}\right)}{6\left(\phi_{A C}-\phi_{A B}\right)} \\
& \text { if }-s\left(\hat{m}_{A} \cdot \boldsymbol{\lambda}\right)=s\left(\hat{m}_{B} \cdot \boldsymbol{\lambda}\right)=-s\left(\hat{m}_{C} \cdot \boldsymbol{\lambda}\right)=\beta
\end{aligned}
$$

In this case it becomes $F=37.5 \%$. Therefore $62.5 \%$ lack of measurement independence for each party is sufficient to simulate statistic of the equatorial Von Neumann measurements on GHZ state.

Acknowledgment: It is a pleasure to thank Guruprasad Kar for many stimulating discussions. MB acknowledge C. Branciard for fruitful discussions. AM acknowledges support from CSIR, Govt. of India (File No.09/093(0148)/ 2012-EMR-I).

\section{References}

1. Einstein, A., Podolsky, B., Rosen, N.: Can Quantum-Mechanical Description of Physical Reality Be Considered Complete? Phys. Rev. 47, 777-780 (1935)

2. Bell, J.S.: On the EINSTEIN-PODOLSKY-ROSEN paradox. Physics 1(3), 195-200 (1964); Bell, J. S.: Speakable and Unspeakable in Quantum Mechanics. Cambridge University Press (2004)

3. Aspect, A., Grangier, P., Roger, G.: Experimental Realization of Einstein-PodolskyRosen-Bohm Gedankenexperiment: A New Violation of Bell's Inequalities. Phys. Rev. Lett. 49, 91-94 (1982)

4. Aspect, A., Dalibard, J., Roger, G.: Experimental Test of Bell's Inequalities Using TimeVarying Analyzers. Phys. Rev. Lett. 49, 1804-1807 (1982)

5. Aspect, A.: Bell's inequality test: more ideal than ever. Nature (London) 398, 189-190 (1999)

6. Greenberger, D.M., Horne, M.A., Zeilinger, A.: Bell's theorem without inequalities. Am J. Phys. 58(12), 1131-1143 (1990)

7. Pan, J.W., Bouwmeester, D., Daniell, M., Weinfurter, H., Zeilinger, A.: Experimental test of quantum nonlocality in three-photon GreenbergerHorneZeilinger entanglement. Nature 403, 515-519 (2000)

8. Brunner, N., Cavalcanti, D., Pironio, S., Scarani, V., Wehner, S.: Bell nonlocality. Rev. Mod. Phys. 86, 419 (2014)

9. Barrett, J., Hardy, L., Kent, A.: No Signaling and Quantum Key Distribution. Phys. Rev. Lett. 95, 010503 (2005)

10. Acín, A., Brunner, N., Gisin, N., Massar, S., Pironio, S., Scarani, V.: Device-Independent Security of Quantum Cryptography against Collective Attacks. Phys. Rev. Lett. 98, 230501 (2007)

11. Pironio, S., Acín, A, Brunner, N., Gisin, N., Massar, S., Scarani, V.: Device-independent quantum key distribution secure against collective attacks. New J. Phys. 11, 045021 (2009)

12. Vazirani, U., Vidick, T.: Fully device independent quantum key distribution. arXiv: 1210.1810

13. Colbeck, R., Kent, A.: Private randomness expansion with untrusted devices. J. Phys. A 44, 095305 (2011)

14. Pironio, S., et al.: Random numbers certified by Bells theorem. Nature (London) 464, 1021-1024 (2010)

15. Banik, M.: Measurement-device-independent randomness certification by local entangled states. arXiv:1401.1338

16. Maudlin, T.: in Proceedings of the 1992 Meeting of the Philosophy of Science Association, edited by D. Hull, M. Forbes, and K. Okruhlik (Philosophy of Science Association, East Lansing, MI). Vol. 1, pp. 404-417 (1992) 
17. Brassard, G., Cleve, R., Tapp, A.: Cost of Exactly Simulating Quantum Entanglement with Classical Communication. Phys. Rev. Lett. 83, 1874-1877 (1999)

18. Steiner, M.: Towards quantifying non-local information transfer: finite-bit non-locality. Phys. Lett. A 270, 239244 (2000)

19. Toner, B. F., Bacon, D.: Communication Cost of Simulating Bell Correlations. Phys. Rev. Lett. 91, 187904 (2003)

20. Tessier, T. E., Caves, C. M., Deutsch I. H., Eastin, B.: Optimal classical-communicationassisted local model of n-qubit Greenberger-Horne-Zeilinger correlations. Phys. Rev. A 72, 032205 (2005)

21. Broadbent, A., Chouha, P. R., Tapp, A.. Proceedings of the Third International Conference on Quantum, Nano and Micro Technologies (ICQNM 2009)

22. Branciard, C., Gisin, N.: Quantifying the Nonlocality of Greenberger-Horne-Zeilinger Quantum Correlations by a Bounded Communication Simulation Protocol. Phys. Rev. Lett. 107, 020401 (2011)

23. Brassard, G., Devroye, L., Gravel, C.: Exact Simulation for the GHZ Distribution. arXiv:1303.5942 (2013)

24. Shimony, A., Horne, M. A., Clauser, J. F.: An exchange on local beables. Dialectica 39, 97-102 (1985)

25. Hall, M. J. W.: Local Deterministic Model of Singlet State Correlations Based on Relaxing Measurement Independence. Phys. Rev. Lett. 105, 250404 (2010)

26. Hall, M. J. W.: Relaxed Bell inequalities and Kochen-Specker theorems. Phys. Rev. A 84, $022102(2011)$

27. Barrett, J., Gisin, N.: How Much Measurement Independence Is Needed to Demonstrate Nonlocality? Phys. Rev. Lett. 106, 100406, (2011)

28. Brans, C. H.: Bell's theorem does not eliminate fully causal hidden variables. Int. J. Theor. Phys. 27(2), 219-226 (1988)

29. Mermin, N. D.: Extreme quantum entanglement in a superposition of macroscopically distinct states. Phys. Rev. Lett. 65, 1838 (1990)

30. Hillery, M., Bužek, V., Berthiaume, A.: Quantum secret sharing. Phys. Rev. A 59, 1829 (1999)

31. Zhao-Yang, T., Le-Man, K.: Broadcasting of Entanglement in Three-Particle Greenberger-Horne-Zeilinger State via Quantum Copying. Chin. Phys. Lett. 17(7), 469$471(2000)$

32. Jin, X-R. et al.: Three-party quantum secure direct communication based on GHZ states. Phys. Lett. A 354(1-2), 67-70 (2006)

33. Banik, M., Gazi, MD R., Das, S., Rai, A., Kunkri, S.: Optimal free will on one side in reproducing the singlet correlation. J. Phys. A: Math. Theor. 45, 205301 (2012) 